\title{
Transverse force on a quantized vortex in a superconductor
}

\author{
Michael R. Geller, ${ }^{1,2}$ Carlos Wexler, ${ }^{1,3}$ and David J. Thouless ${ }^{1}$ \\ ${ }^{1}$ Department of Physics, University of Washington, Seattle, Washington 98195 \\ ${ }^{2}$ Department of Physics and Astronomy, University of Georgia, Athens, Georgia 30602 \\ ${ }^{3}$ Department of Physics, University of Florida, Gainesville, FL 32611
}

(September 12, 2018)

\begin{abstract}
The total transverse force acting on a quantized vortex in a type-II superconductor determines the Hall response in the mixed state, yet a consensus as to its correct form is still lacking. In this paper we present an essentially exact expression for this force, valid in the superclean limit, which was obtained by generalizing the recent work by Thouless, Ao, and Niu [D. J. Thouless, P. Ao, and Q. Niu, Phys. Rev. Lett. 76, 3758 (1996)] on the Magnus force in a neutral superfluid. We find the transverse force per unit length to be $\mathbf{f}=\rho \mathbf{K} \times \mathbf{V}$, where $\rho=\rho_{\mathrm{n}}+\rho_{\mathrm{s}}$ is the sum of the mass densities of the normal and superconducting components, $\mathbf{K}$ is a vector parallel to the line vortex with a magnitude equal to the quantized circulation, and $\mathbf{V}$ is the vortex velocity.
\end{abstract}

PACS: 74.60.Ge, 47.37.+q, 67.40.Vs

The discovery of high-temperature superconductivity has stimulated a renewed interest in the statistical mechanics and dynamics of vortices in type-II superconductors [1]. Despite the tremendous progress made, however, the answer to one of the simplest questions, namely, the form of the equation of motion for a single isolated vortex, remains controversial. If we let $\mathbf{R}(t)$ denote the position in the $x y$ plane of the center of a straight vortex line as a function of time, then the classical equation of motion can be written as

$$
M \frac{d^{2} \mathbf{R}}{d t^{2}}=-\eta \frac{d \mathbf{R}}{d t}-\gamma \frac{d \mathbf{R}}{d t} \times \mathbf{e}_{z}+\mathbf{f}_{\mathrm{p}}(\mathbf{R})+\mathbf{f}_{\mathrm{d}}(\mathbf{R}) .
$$

Here we have taken the circulation vector $\mathbf{K}$ of the vortex (a vector parallel to the vortex with a magnitude equal to the circulation (2]) to be along the $z$ direction. The first two terms on the right-hand-side of (11) are to include all forces linear in the vortex velocity. Here $\mathbf{f}_{\mathrm{p}}$ represents the pinning force due to disorder (averaged over an area set by the size of the vortex core), and $\mathbf{f}_{\mathrm{d}}$ includes the various "driving" forces possible, such as the Lorentz force, which may depend on the density of the normal and superconducting components $n_{\mathrm{s}}$ and $n_{\mathrm{n}}$, and therefore on the vortex position $\mathbf{R}$, but do not depend on the vortex velocity. The equation of motion (1) also describes a vortex in a Bose or Fermi superfluid, where $\mathbf{f}_{\mathrm{p}}$ might describe the force from an externally imposed wire as in a Vinen-like experiment [3], and $\mathbf{f}_{\mathrm{d}}$ would include the superfluid-velocity-dependent part of the Magnus force [4] and possibly other vortex-velocity-independent contributions [5].

The difficulty concerns the determination of the coefficients $M, \eta$, and $\gamma$, which describe the vortex effective mass per unit length, viscous damping force per unit length, and nondissipative transverse force per unit length, respectively. Of particular interest and especially controversial is the coefficient $\gamma$, which in a neutral superfluid has been recently shown by Thouless, Ao, and Niu [6] (hereafter referred to as TAN) to be $\gamma=\rho_{\mathrm{s}} K$, where
$\rho_{\mathrm{S}}$ is the mass density of the superfluid component far from the vortex core, and $K$ is the quantized circulation. For a vortex in a neutral Bose superfluid with $q$ quanta of circulation, this coefficient is simply $\gamma=q h n_{\mathrm{s}}$, with $n_{\mathrm{s}}$ the superfluid number density away from the core, whereas in a (paired) Fermi superfluid $\gamma=\frac{1}{2} q h n_{\mathrm{s}}$. The TAN result shows that the transverse force is universal, independent of the detailed microscopic structure of the vortex and its interaction with the normal component of the fluid. The universality of the nondissipative transverse force is not found in some approximate microscopic calculations, however, which obtain coefficients that reduce to the TAN result only in certain limits [7 9].

In this paper we calculate the nondissipative transverse force on a quantized vortex in a charged superfluid or superconductor by following the method of TAN [6]. Initially, we shall consider a system with translational invariance, and to address a more realistic model of a superconductor we include afterwards a periodic potential from the lattice, assuming the lattice constant $b$ is small compared with the coherence length $\xi$. In both cases we find the transverse force per length to be given by

$$
\mathbf{f}=\rho \mathbf{K} \times \mathbf{V},
$$

where $\rho=\rho_{\mathrm{n}}+\rho_{\mathrm{s}}$ is the total mass density of the fluid away from the core, $\mathbf{K}$ is the quantized circulation vector defined above, and $\mathbf{V}$ is the constant vortex velocity. Most relevant is the fact that, as in TAN, our results for this superclean limit of a superconductor also indicate a universal vortex-velocity-dependent part of the transverse force. In the notation of Eqn. (11), we find $\gamma=\rho K$. For a vortex with $q$ trapped flux quanta $\Phi_{0} \equiv h c / 2 e$ we can write this as $\gamma=\frac{1}{2} q h n$, where $n$ is the total density of the fluid far from the core. The reason that the transverse force in the charged case differs from that for the neutral superfluid, which has a coefficient $\rho_{\mathrm{s}}$ instead of $\rho$, is related to the Meissner effect, as will be explained below. 
The Hamiltonian for the charged superfluid or superconductor is taken to be

$$
H=H_{0}+H_{1}+\sum_{n} V\left(\mathbf{r}_{n}-\mathbf{R}\right),
$$

where

$$
H_{0} \equiv \sum_{n} \frac{p_{n}^{2}}{2 m}+\frac{1}{2} \sum_{n \neq n^{\prime}} U\left(\mathbf{r}_{n}-\mathbf{r}_{n^{\prime}}\right)+H_{\mathrm{b}}
$$

is the Hamiltonian for $N$ bosons or fermions with negative charge $-e$, interacting with each other and with a uniform positive background charge, and (in Gaussian units)

$$
H_{1} \equiv-\frac{e^{2}}{2 m^{2} c} \sum_{n \neq n^{\prime}} p_{n}^{i} T^{i j}\left(\mathbf{r}_{n}-\mathbf{r}_{n^{\prime}}\right) p_{n^{\prime}}^{j}
$$

with $T^{i j}(\mathbf{r}) \equiv\left(\delta^{i j}|\mathbf{r}|^{-1}+r^{i} r^{j}|\mathbf{r}|^{-3}\right) / 2 c$, is the currentcurrent interaction, which, as first noted by Darwin [10], correctly accounts for the electrodynamics in the transverse gauge through order $v^{2} / c^{2}$. The $U$ in (4) is a Coulomb interaction term, and in the case of a superconductor also contains an additional short-ranged attractive interaction to produce superconductivity. Also, $H_{\mathrm{b}} \equiv-\sum_{n} \int d^{3} r e^{2} \bar{n}\left|\mathbf{r}_{n}-\mathbf{r}\right|^{-1}$ accounts for the interaction with the uniform positive background charge $e \bar{n}$. The full Hamiltonian also contains a cylindrically symmetric pinning potential $V$ centered at position $\mathbf{R}$ 11].

Before proceeding it is important to note that the Hamiltonian (3), with the current-current interaction term, provides an accurate model of a charged superfluid or superconductor. In particular, we show in the appendix that it exhibits a Meissner effect with the conventional London screening length.

We shall follow TAN and calculate the force on the vortex by expanding the time-dependent wave function, given by $i \hbar \partial_{t}|\Psi(t)\rangle=H(\mathbf{R}(t))|\Psi(t)\rangle$, in a basis of instantaneous eigenstates satisfying $H(\mathbf{R})\left|\psi_{\alpha}(\mathbf{R})\right\rangle=$ $E_{\alpha}(\mathbf{R})\left|\psi_{\alpha}(\mathbf{R})\right\rangle$. We shall assume a three-dimensional system with the $z$ direction along $\mathbf{K}$. The system is assumed to be infinite in the $x$ and $y$ directions, and $L$ is the thickness in the $z$ direction, which is also the length of the vortex. In the absence of the pinning potential the system is therefore translationally invariant in the $x$ and $y$ directions. The translational invariance allows the instantaneous eigenfunctions to be taken as $\psi_{\alpha}^{\mathbf{R}}\left(\mathbf{r}_{1}, \cdots, \mathbf{r}_{N}\right)=\psi_{\alpha}\left(\mathbf{r}_{1}-\mathbf{R}, \cdots, \mathbf{r}_{N}-\mathbf{R}\right)$, where $\psi_{\alpha}\left(\mathbf{r}_{1}, \cdots, \mathbf{r}_{N}\right)$ are the eigenfunctions with the pinning potential centered at the origin. Initially, at a time $t_{0}$, a vortex is assumed to be bound to the pinning potential at $\mathbf{R}\left(t_{0}\right)=\mathbf{R}_{0}$ in a state characterized by the density matrix $\sum_{\alpha} f_{\alpha}\left|\psi_{\alpha}\left(\mathbf{R}_{0}\right)\right\rangle\left\langle\psi_{\alpha}\left(\mathbf{R}_{0}\right)\right|$, where $f_{\alpha}$ is the occupation probability of state $\alpha$. The force on the vortex is $\mathbf{F}=-\sum_{\alpha} f_{\alpha}\left\langle\Psi_{\alpha}(t)\left|\nabla_{R} H\right| \Psi_{\alpha}(t)\right\rangle$, where $\left|\Psi_{\alpha}(t)\right\rangle$ is the solution of the time-dependent Schrödinger equation starting out in $\left|\psi_{\alpha}\left(\mathbf{R}\left(t_{0}\right)\right)\right\rangle$. Following Ref. [6] we obtain a transverse force equal to

$$
\mathbf{F}=\left.\frac{i \hbar L}{2}\left(\mathbf{e}_{z} \times \mathbf{V}\right) \oint_{a} d \mathbf{l} \cdot\left(\nabla-\nabla^{\prime}\right) \rho\left(\mathbf{r}, \mathbf{r}^{\prime}\right)\right|_{\mathbf{r}^{\prime} \rightarrow \mathbf{r}},
$$

where $\rho\left(\mathbf{r}, \mathbf{r}^{\prime}\right)$ is the one-particle density matrix and $L$ is the thickness of the system in the $z$ direction. In (6) we choose the radius $a$ of the integration contour to be much larger than the London penetration depth $\lambda$.

The integrand in (6) is proportional to the canonical momentum density. The current-current interaction term (5) has played no role up to this point, and, indeed, the expression (6) in the two-dimensional limit is identical to that obtained by TAN for the neutral superfluid. However, the resulting force is different, as can be seen by writing (6) in terms of the canonical momentum density, which leads to a transverse force per unit length equal to

$$
\mathbf{f}=\mathbf{e}_{z} \times \mathbf{V} \oint_{a} d \mathbf{l} \cdot \mathbf{j}(\mathbf{r}) .
$$

The TAN result for neutral superfluids is obtained by writing the momentum density as $\mathbf{j}=\rho_{\mathrm{n}} \mathbf{v}_{\mathrm{n}}+\rho_{\mathrm{s}} \mathbf{v}_{\mathrm{s}}$ and assuming that the viscous normal component does not circulate 12. In the case of a charged superfluid or superconductor, however, the gauge-invariant momentum density $\mathbf{j}+\frac{e}{c} n \mathbf{A}$ vanishes in the region containing the integration contour because of the Meissner effect (see Appendix), and we therefore obtain (2).

Our conclusion suggests that both the normal and superconducting components of the fluid contribute to the total transverse force. However, we would like to emphasize that the model considered here does not include disorder, and, as such, leads to a normal component having an infinite conductivity. This neglect of disorder may be responsible for the $\rho$ dependence of the transverse force instead of the usual $\rho_{\mathrm{s}}$.

Finally, we would like to remark that the result (2) holds even in the presence of a lattice, as long as the coherence length or vortex core size $\xi$ is large compared with the lattice constant $b$. To demonstrate this we add a periodic potential term $\sum_{n} v\left(\mathbf{r}_{n}\right)$ to $H_{0}$ and expand the instantaneous eigenstates in a basis of localized Wannier functions $a(\mathbf{r}-\mathbf{l})$ for the relevant band,

$$
\begin{aligned}
\psi_{\alpha}^{\mathbf{R}}\left(\mathbf{r}_{1}, \cdots, \mathbf{r}_{N}\right) & =\sum_{\mathbf{l}_{1} \cdots \mathbf{l}_{N}} \phi_{\alpha}^{\mathbf{R}}\left(\mathbf{l}_{1}, \cdots, \mathbf{l}_{N}\right) a\left(\mathbf{r}_{1}-\mathbf{l}_{1}\right) \\
& \times a\left(\mathbf{r}_{2}-\mathbf{l}_{2}\right) \cdots a\left(\mathbf{r}_{N}-\mathbf{l}_{N}\right)
\end{aligned}
$$

Here $\mathbf{l}$ labels the sites of the lattice, and the coefficients $\phi_{\alpha}^{\mathbf{R}}\left(\mathbf{l}_{1}, \cdots, \mathbf{l}_{N}\right)$ are taken to be completely symmetric or antisymmetric. Assuming that we are at the minimum of a band with an isotropic effective mass $m^{*}$, we see that the envelope function $\phi_{\alpha}^{\mathbf{R}}\left(\mathbf{r}_{1}, \cdots, \mathbf{r}_{N}\right)$ satisfies a Schrödinger equation with a Hamiltonian given by (3) apart from the replacement of $m$ in $H_{0}$ with $m^{*}$ (the mass in $H_{1}$ is not changed). This is the standard effective mass approximation, and it leads to 


$$
\mathbf{F}=\left.\frac{i \hbar}{2}\left(\mathbf{e}_{z} \times \mathbf{V}\right) \int_{0}^{L} d z \oint_{a} d \mathbf{l} \cdot\left(\nabla-\nabla^{\prime}\right) \rho_{\mathrm{eff}}\left(\mathbf{r}, \mathbf{r}^{\prime}\right)\right|_{\mathbf{r}^{\prime} \rightarrow \mathbf{r}},
$$

where

$$
\begin{aligned}
\rho_{\mathrm{eff}}\left(\mathbf{r}, \mathbf{r}^{\prime}\right) & \equiv N \sum_{\alpha} f_{\alpha} \int d^{3} r_{2} d^{3} r_{3} \cdots d^{3} r_{N} \\
& \times \phi_{\alpha}^{*}\left(\mathbf{r}, \mathbf{r}_{2}, \cdots, \mathbf{r}_{N}\right) \phi_{\alpha}\left(\mathbf{r}^{\prime}, \mathbf{r}_{2}, \cdots, \mathbf{r}_{N}\right)
\end{aligned}
$$

is a one-particle density matrix constructed from the envelope functions.

Unfortunately, the integrand in (9) is not proportional to the actual canonical momentum density $\mathbf{j}(\mathbf{r})$, the latter having fluctuations on the scale of the lattice constant $b$. However, it is possible to prove that when any local single-particle operator like the current density is averaged over a length scale larger than the localization length of the Wannier functions (assumed to be of the order of $b$ ), but smaller than the characteristic length scale over which the envelope functions vary, the expectation value is correctly given by $\rho_{\text {eff }}\left(\mathbf{r}, \mathbf{r}^{\prime}\right)$ 13]. In other words, the structure on the scale of the lattice constant is not described correctly by the envelope functions, but coarse-grained quantities are. Now, because the line integral of the actual canonical momentum is quantized, it is possible to write it as

$$
\oint_{a} d \mathbf{l} \cdot \mathbf{j}=\frac{1}{a_{2}-a_{1}} \int_{a_{1}}^{a_{2}} d a \frac{1}{L} \int_{0}^{L} d z \oint_{a} d \mathbf{l} \cdot \mathbf{j}
$$

where both radii $a_{1}$ and $a_{2}$ are much larger than $\lambda$ and their difference is larger than $b$. The integrals over $a$ and $z$ on the right-hand-side of (11) have the effect of averaging the azimuthal component of $\mathbf{j}$. Therefore, the actual circulation is correctly given by the line integral of the coarse-grained momentum, which, in turn, is correctly given by the envelope functions. Hence, (9) leads to the transverse force (2), as stated.

The total transverse force per unit length on the vortex may be obtained by adding the conventional Lorentz force term $\mathbf{f}_{\mathrm{L}}=-\frac{e}{c} n \mathbf{v}_{\mathrm{e}} \times \mathbf{\Phi}$, leading to

$$
\mathbf{f}=\rho \mathbf{K} \times\left(\mathbf{V}-\mathbf{v}_{\mathrm{e}}\right)=\frac{e}{c} \int d^{2} r n\left(\mathbf{V}-\mathbf{v}_{\mathrm{e}}\right) \times \mathbf{B} .
$$

Alternatively, we can write this as

$$
\mathbf{f}=\frac{e}{c} \int d^{2} r n\left(\mathbf{v}_{\mathrm{p}}-\mathbf{v}_{\mathrm{e}}\right) \times \mathbf{B}+\frac{e}{c} \int d^{2} r n\left(\mathbf{V}-\mathbf{v}_{\mathrm{p}}\right) \times \mathbf{B},
$$

where $\mathbf{v}_{\mathrm{p}}$ is the velocity of the positively charged substrate, usually taken to be at rest. The first term in (13) is the Lorentz force, given by the interaction of the Galilean-invariant current with the magnetic field, while the second term is a Magnus force that acts on the substrate. This interpretation is in agreement with the early picture of flux line motion in type-II superconductors given by Nozières and Vinen in the late sixties [14]: The Magnus force on the vortex may be thought as the Kutta-Joukowski hydrodynamic lift force due to the circulation of the electron fluid around the vortex. Far from the vortex the circulation is reduced but an increase in the Lorentz force exactly compensates this deficiency. The Magnus force reaction is eventually carried away by the positive substrate.

This work was supported by the NSERC of Canada and by the NSF through grant No. DMR-9528345. M. G. would like to acknowledge the kind hospitality of the Department of Physics at the University of Washington where this work was done.

\section{APPENDIX A: MEISSNER EFFECT IN THE RPA APPROXIMATION}

As mentioned above, it is important to establish that the Darwin Hamiltonian provides an accurate model of a charged superfluid or superconductor; in particular, that it exhibits a Meissner effect with the conventional London penetration depth.

This can be demonstrated by treating the currentcurrent interactions in an RPA-type approximation and calculating the current induced by a weak applied vector potential,

$$
J^{i}(\mathbf{q})=\chi^{i j}(\mathbf{q}) A_{\mathrm{ext}}^{j}(\mathbf{q}) .
$$

The zero-frequency linear response function $\chi^{i j}$ is the sum of a retarded current-current correlation function $\Pi_{\mathrm{R}}^{i j}$ for a system described by the Hamiltonian $H_{0}+H_{1}$, and a diamagnetic term. Our RPA approximation corresponds to a summation of the diagrams shown in Fig. 11 for the imaginary-time current-current correlation function $\Pi^{i j}\left(i \omega_{n}, \mathbf{q}\right)$, which are the most divergent terms as $\mathbf{q} \rightarrow 0$. In this approximation we find

$$
\chi=\chi_{0}+\chi_{0} T \chi
$$

where $\chi_{0}^{i j}$ is the corresponding response function for the system without current-current interactions, as described by $H_{0}$, and $T^{i j}(\mathbf{q})=4 \pi\left(\delta^{i j}|\mathbf{q}|^{-2}-q^{i} q^{j}|\mathbf{q}|^{-4}\right) / c$ is the Fourier transform of $T^{i j}(\mathbf{r})$.

The response function $\chi^{i j}$ can be used to relate the total vector potential $\mathbf{A}_{\text {tot }} \equiv \mathbf{A}_{\text {ext }}+\mathbf{A}_{\text {ind }}$, the sum of an external and induced part, to $\mathbf{A}_{\text {ext }}$ itself, 


$$
A_{\mathrm{tot}}^{i}(\mathbf{q})=\left[1-T(\mathbf{q}) \chi_{0}(\mathbf{q})\right]_{i j}^{-1} A_{\mathrm{ext}}^{j}(\mathbf{q}) .
$$

Because $\omega=0$ here, (A3) tells us about magnetic screening. Now, $\chi_{0}^{i j}$ is known to have some general properties in the $\mathbf{q} \rightarrow 0$ limit, reflecting the presence of off-diagonal long-range order. In particular,

$$
\chi_{0}^{i j}(\mathbf{q})=\frac{n_{\mathrm{s}} e^{2}}{m c}\left(\delta^{i j}-q^{i} q^{j} /|\mathbf{q}|^{2}\right)
$$

in this limit [15], resulting in

$$
\mathbf{A}_{\text {tot }}(\mathbf{q})=\left(1+\frac{1}{\lambda^{2} q^{2}}\right)^{-1} \mathbf{A}_{\mathrm{ext}}(\mathbf{q})
$$

where $\lambda \equiv\left(m c^{2} / 4 \pi n_{\mathrm{s}} e^{2}\right)^{\frac{1}{2}}$ is the usual London penetration depth. Thus, the current-current interaction term leads to a conventional Meissner effect.

[1] G. Blatter, M. V. Feigel'man, V. B. Geshkenbein, A. I. Larkin, and V. M. Vinokur, Rev. Mod. Phys. 66, 1125 (1994).

[2] We define the circulation generally as $K \equiv \oint(\mathbf{j} / \rho) \cdot d \mathbf{l}$, where $\rho(\mathbf{r})$ is the mass density and $\mathbf{j}(\mathbf{r})$ is the canonical momentum density $\mathbf{j}(\mathbf{r}) \equiv\left\langle\operatorname{Re} \psi^{\dagger}(\mathbf{r}) \frac{\hbar}{i} \nabla \psi(\mathbf{r})\right\rangle$. In a Bose superfluid $K$ is quantized in positive integer multiples of $h / m$, with $m$ the particle mass, whereas in a Fermi superfluid or superconductor it is quantized in multiples of $h / 2 m$.

[3] W. F. Vinen, Proc. R. Soc. London A 260, 218 (1961).

[4] C. Wexler, Phys. Rev. Lett. 79, 1321 (1997).

[5] E. B. Sonin, Phys. Rev. B 55, 485 (1997).

[6] D. J. Thouless, P. Ao, and Q. Niu, Phys. Rev. Lett. 76, 3758 (1996).

[7] N. B. Kopnin and V. E. Kravtsov, Sov. Phys. JETP 44, 861 (1976). N. B. Kopnin and M. M. Salomaa, Phys. Rev. B 44, 9667 (1991). N. B. Kopnin, G. E. Volovik, and Ü. Parts, Europhys. Lett. 32, 651 (1995).

[8] G. E. Volovik, Sov. Phys. JETP 77, 435 (1993); JETP Lett. 62, 66 (1995).

[9] A. van Otterlo, M. V. Feigel'man, V. B. Geshkenbein, and G. Blatter, Phys. Rev. Lett. 75, 3736 (1995).

[10] J. D. Jackson, Classical Electrodynamics (John Wiley and Sons, New York, 1975).

[11] The other order $v^{2} / c^{2}$ relativistic corrections do not affect our result (2).

[12] For further discussion, see C. Wexler and D. J. Thouless, cond-mat/9612059.

[13] As a simple example, consider the density $n(\mathbf{r})=$ $|\psi(\mathbf{r})|^{2}=\sum_{\mathbf{l l}^{\prime}} \phi^{*}(\mathbf{l}) \phi\left(\mathbf{l}^{\prime}\right) a(\mathbf{r}-\mathbf{l}) a\left(\mathbf{r}-\mathbf{l}^{\prime}\right)$ for one electron in a crystal. After coarse-graining, $\overline{n(\mathbf{r})}=|\phi(\mathbf{r})|^{2}$.

[14] P. Nozières and W. F. Vinen, Phil. Mag. 14, 667 (1966).

[15] J. R. Schrieffer, Theory of Superconductivity (AddisonWesley, New York, 1988).

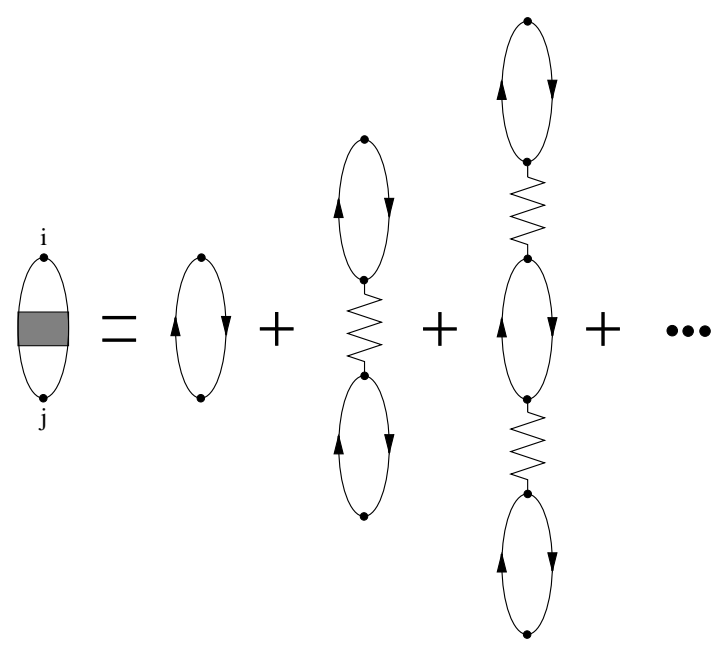

FIG. 1. RPA approximation for the correlation function $\Pi^{i j}\left(i \omega_{n}, \mathbf{q}\right)$. Here the solid lines denote the exact Green's functions for $H_{0}$ and the zig-zag lines represent the current-current interaction $T^{i j}(\mathbf{q})$. 